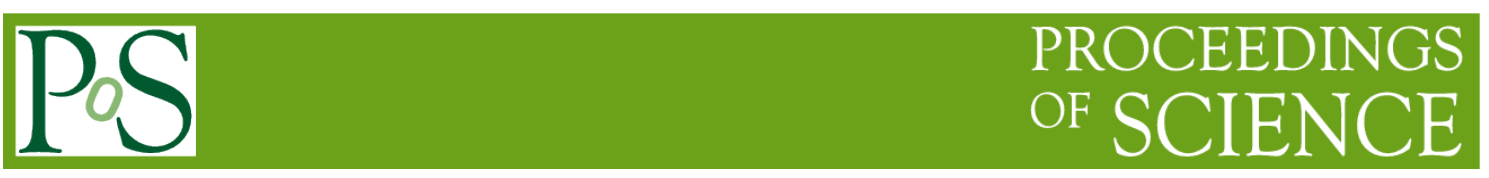

\title{
Research into Radar Net Anti-interference Strategy Based on Improved Genetic Algorithm
}

\author{
Xuekui Wang , Xiaoyan Yang , Huiling Chen \\ Air Defense Forces Academy \\ E-mail:zhzhwxk@163.com
}

Under complicated electromagnetism environment, it is effective to raise the effect of antiinterference by networking the radar sets of different frequencies and systems. This paper built the nonlinear programming model of quantitative analysis by researching the problems of radar net anti-interference effect, and blended the thought of simulated annealing algorithm with genetic algorithm for improvement to solve models and give the anti-interference strategies of radar net. 


\section{Introduction}

On the informatization battlefield, as the electronic combat becomes the guide and runs through the war from beginning to end, it is especially important for the radar to possess the ability of anti-interference. Particularly in the complicated electromagnetism environment, radars must feature stronger anti-interference ability if they want to exist [1]. Currently, researches on the anti-interference strategies of netted radars are relatively less. This paper built the anti-interference strategy model of radar net on the foundation of quantitative analysis to seek the strategy solution based on the improved genetic algorithm.

\section{Establishment of Radar Net Anti-Interference Strategy Model}

\subsection{Model Analysis}

The interference modes of the enemy's interference equipment in the air defense combat are usually diversified as follows: barrage, transmitting deception, spotting, scanning and chaff. Supposing our radar net be composed of three radar sets of different systems, among them, Radar A can adopt the anti-interference strategy of frequency agility, amplitude limiting-stable false alarm, silent sector and power resisting, etc.; Radar B can adopt the anti-interference strategy of the automatic frequency choice and wideband dicke-fix and sidelobes cancellation against each other and sidelobe hiding, etc [2].; Radar C can adopt the anti-interference strategy of anti-wideband dicke-fix, frequency agility and so forth in response to the interference style of enemy.

Given the anti-interference strategy set of our radar net as

$$
S_{k}=\left\{a_{1}^{k}, a_{2}^{k}, \ldots, a_{n_{k}}^{k}\right\}
$$

Given the anti- interference strategy set of enemy as

$$
S_{2}=\left\{b_{1}, b_{2}, \ldots, b_{m}\right\}
$$

There in

$k=1,2,3$ refers to the radars of different systems;

$n_{k}$ refers to the anti-interference style of number $k$;

$a_{j}^{k}$ refers to the anti- interference style of type $j$ in radar $k$;

$m$ refers to the number of the anti-interference styles.

\subsection{Model Establishment}

The won matrix of the anti-interference strategy model of our radar net from analysis is

$$
\begin{gathered}
A=\left\{A_{1}^{T}, A_{2}^{T}, A_{3}^{T}\right\}^{T} \\
A_{k}=\left\{\begin{array}{cccc}
a_{11}^{k} & a_{12}^{k} & \ldots & a_{15}^{k} \\
a_{21}^{k} & a_{22}^{k} & \ldots & a_{25}^{k} \\
\ldots a_{n 1}^{k} & a_{n 2}^{k} & \ldots & a_{n 5}^{k}
\end{array}\right\}
\end{gathered}
$$

$A_{k}$ refers to the won matrix of radar $k$ in radar net;

Matrix element $A_{i j}^{k}$ stands for he probability of discovering the target of radar $k$ by adopting anti-interference strategy $i$ in interference mode $j$

Given $X=\left\{X_{1}, X_{2}, X_{3}\right\}$ is the comprehensive strategy of radar net; 
Therein $\quad X_{k}=\left\{X_{1}^{k}, X_{2}^{k}, X_{3}^{k}\right\}$ is the mixed strategy of radar ;

Given the enemy strategy is $Y=\left\{Y_{1}, Y_{2}, Y_{3}\right\}$, the probability of radar's discovering target in enemy interference is $v_{k}$, then the probability of all radars'discovering target in radar net is $P_{1}=\prod_{k} v_{k} \quad$ [3], but the probability of radar net's ability to discover the target is $P_{2}=1-\prod_{k}\left(1-v_{k}\right)$.

Then the optimal strategy on our part is to make it possible of discovering the target in radar net maximum, that is, to make $p_{2}$ to reach the maximum while making the arbitrary radar's probability of discovering as the minimum of probability of discovering, that is $\sum_{i} a_{i j}^{k} x_{i}^{k} \geqslant v_{k}$

Similarly, with their whole interference in the radar net,the enemy is to make probability of discovering target of the radar net to be wholly lowest,that is, $P_{1}$ reaches the minimum, $\sum_{i} a_{i j}^{k} y_{j} \leqslant v_{k}$ fulfillingat meanwhile.

Thus, the optimal strategic solution is non-linear programming problem(2.5) [4]:

$$
\begin{gathered}
\operatorname{Max} \prod_{k=1}^{3} v_{k} \\
\sum_{i} a_{i j}^{k} x_{i}^{k} \geqslant v_{k} \\
\sum_{i} x_{i}^{k}=1 \\
x_{i}^{k} \geqslant 0
\end{gathered}
$$

$$
\begin{gathered}
k=1,2,3 \\
i=1, \ldots, n_{1}+n_{2}+n_{3} \\
j=1, \ldots, 5
\end{gathered}
$$

The optimal strategic solution of enemy is non-linear programming problem(2.6):

$$
\begin{array}{cc}
\operatorname{Min} 1-\prod_{k=1}^{3}\left(1-v_{k}\right) & \\
\sum_{j} a_{i j}^{k} y_{j} \leqslant v_{k} & k=1,2,3 \\
\sum_{j} y_{j}=1 & i=1, \ldots, n_{1}+n_{2}+n_{3} \\
y_{j} \geqslant 0 & j=1, \ldots, 5
\end{array}
$$

\section{Model Solution of the Improved Genetic Algorithm}

Formula(2.5) and(2.6) are the optimal strategic solution model based on non-linear programming, which is more complicated. We usually use particle swarm optimization (PSO), simulated annealing algorithm and genetic algorithm (GA) for solution [3]. There is a problem of premature convergence in the algorithm of PSO itself and it doesn't have the mechanism of dealing with the constrain condition. Although the simulated annealing algorithm has a stronger search ability, it is easily trapped in the local optimum. Comparatively speaking, the genetic algorithm has the characteristic of good overall optical search. This paper carries on the solution by mixing these two algorithms of simulated annealing and genetic ones to develop the local optimum ability, which can achieve sound analysis of optical result in the computer programming. 


\subsection{Code Scheme}

The coding of genetic algorithm is generally encoded in binary system but requires a higher accuracy to solve the strategy problem [5]. We can solve it by magnifying each strategy value by 100,000 times, so the string length of the binary system codes is very big and we should adopt the decimal system coding.

Take Equation(2.5) as an example and make the stipulation condition transformed as follows:

$$
\begin{gathered}
v_{k}={ }_{j} \operatorname{Min}_{i} a_{i j}^{k} x_{i}^{k} \\
0 \leqslant x_{1} \leqslant 100000 \\
0 \leqslant x_{2} \leqslant 100000 \\
x_{3}=100000-\sum_{s=1}^{2} x_{s}
\end{gathered}
$$

As known from the analysis, we only need to code the first two decision variables. can be decided by other variables.

\subsection{Chromosomes Evaluation}

Firstly, the chromosomes are randomly generated. After the numbers in $[0,1]$ are magnified to 100,000 times, we can evaluate the penalty function and construct the penalty function.

$$
g_{k}(x)=\left\{\begin{array}{cc}
1 & x_{i} \text { meets all the conditions of equation } 3.1 \\
a t_{k} & x_{i} \text { doesn't meet the condition of miminum value } \\
0 & x_{3}<0
\end{array}\right\}
$$

Therein, $\quad a$ is a random number among $[0,1]$ to control the choice of chromosomes.

$t_{k}$ refers to the cooling coefficient in simulated annealing algorithm like this. The coefficient generates cooling once as the genetic algorithm cycles once, so the cooling coefficient of the $(\mathrm{k}+1)^{\text {th }}$ cycle is $t_{k+1}=b t_{k} \quad b \in(0,1)$ 。

When the first two coefficients are larger to make $x_{3}<0$, we should give up this set of coefficients, so the penalty function is 0 now.

\subsection{Fitness Function}

In the calculation methods, the fitness function should be related to the target function value and the penalty function. The more optimal the target function is, the larger the fitness function will be; so the fitness function is:

$$
G(x)=g(x) * \prod_{k=1}^{3} v_{k}
$$

\subsection{Genetic Operation}

In combination with the simulated annealing algorithm with the genetic algorithm, we can adopt the following steps:

1) Selection

The selection implies the adoption of mixed strategies of fitness-proportionate method and elite reservation to decide the selective probability. First, direct duplication of the individual 
with highest fitness in the population into the next generation community, then select the population left with the probability based on the size of fitness function.

2) Cross

The cross means the adoption of the algorithm across two points. In the two paired individual codes, we can choose two crossing points randomly and exchange the codes of crossing points each other to get a new individual.

3)Variation

The variation is to adopt the self-adaptation mutation algorithm. The mutation probability adjusts the self-adaptively as the variation trend of the population, the size of mutation probability is relevant to the difference $\nabla(k)$ between the new individual and the old one in the $k^{\text {th }}$ genetic cycling. The larger the difference is, the smaller the mutation probability will be, and the mutation probability is :

$$
P_{k}=\frac{1}{\left(1+e^{(\nabla(k))}\right)}
$$

4)Terminate

This algorithm is a method that employs controlling the number of cycling times, generally controlled in 1000 times.

The flowchart of the algorithm is as follows:

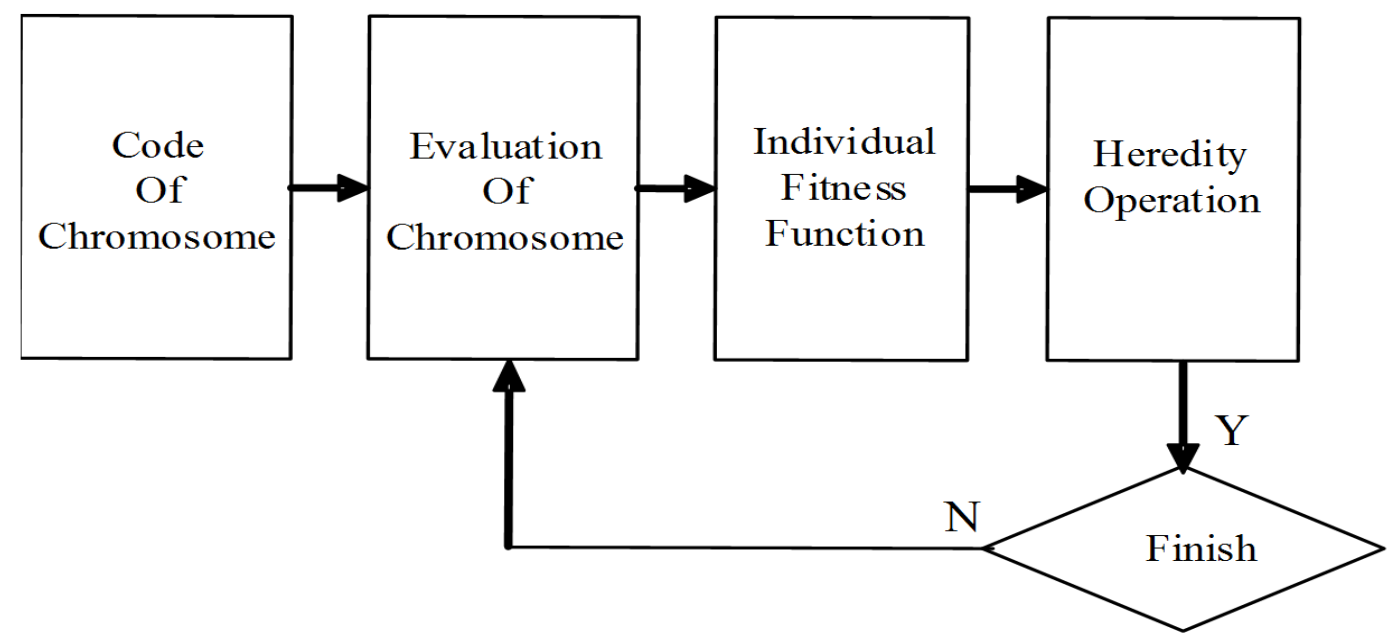

Chart 1: Process of Genetic Algorithm

It's omitted here about the operation of Equation(2.6), which is the same.

\section{Analysis of Examples}

Suppose that the style of the enemy interference and the anti-interference strategy of our radar net be described as 2.1, the probability of each radar's discovering the target is shown in Table 1. 


\begin{tabular}{|c|ccccc|}
\hline & $b_{1}$ & $b_{2}$ & $b_{3}$ & $b_{4}$ & $b_{5}$ \\
\hline$a_{1}^{1}$ & 0.86 & 0.72 & 0.07 & 0.04 & 0.84 \\
\hline$a_{2}^{1}$ & 0.33 & 0.70 & 0.45 & 0.54 & 0.04 \\
\hline$a_{1}^{2}$ & 0.66 & 0.78 & 0.72 & 0.54 & 0.58 \\
\hline$a_{2}^{2}$ & 0.46 & 0.76 & 0.04 & 0.73 & 0.47 \\
\hline$a_{3}^{2}$ & 0.79 & 0.28 & 0.24 & 0.56 & 0.73 \\
\hline$a_{4}^{2}$ & 0.66 & 0.51 & 0.27 & 0.52 & 0.65 \\
\hline$a_{1}^{3}$ & 0.56 & 0.73 & 0.24 & 0.74 & 0.81 \\
\hline$a_{2}^{3}$ & 0.05 & 0.72 & 0.65 & 0.43 & 0.44 \\
\hline$a_{3}^{3}$ & 0.62 & 0.51 & 0.37 & 0.25 & 0.41 \\
\hline$a_{4}^{4}$ & 0.34 & 0.71 & 0.31 & 0.57 & 0.39 \\
\hline
\end{tabular}

Table 1:Probability Matrix of Target Discovery by the Radar Net

After the cycling calculation of 500 times, our strategy of radar net is

$$
X_{1}=(0.30,0.70,0,0) \quad, \quad X_{2}=(0.76,0.24,0,0) \quad, \quad X_{3}=(0.10,0.42,0.48,0)
$$

The probability of discovering the enemy by the radar net is 0.80 .

In comparison of different algorithms above, it can be seen that we can get the optimal strategy with the mixing algorithms in this paper.

\begin{tabular}{|c|ccc|}
\hline Pteration times & \multirow{2}{*}{ PSO } & GA & Mixing GA \\
\hline 100 & 0.741 & 0.712 & 0.747 \\
\hline 300 & 0.780 & 0.720 & 0.790 \\
\hline 500 & 0.780 & 0.724 & 0.796 \\
\hline
\end{tabular}

Table 2: Comparison Matrix of Different Algorithms

Similarly, the strategy of the enemy interference equipment is $Y=(0.30,0,0.49,0.13,0.08)$ :

The probability of discovering the target simultaneously by three radars is 0.08 .

\section{Conclusion}

This paper is to solve the problem of radar net's anti-interference strategy based on the genetic algorithm. Based on the analysis, we can make it more possible of radars net to discover the target with reasonable anti-interference strategy, which may improve the fighting efficiency of the radar net. The model solution is to adopt the annealing thought of simulated annealing algorithm to carry out chromosomes evaluation and genetic operation, which can quicken the speed of solution effectively and get overall optimum in practical application. 


\section{References}

[1] Sun Guozhi, “Electron Combat”, Press of Military Science, 2009.

[2] Zhao Guoqing. "Theory of Radars confronting"[M].Press of Xi' an Electronic and EngineeringUniversity.1999.

[3] Jiang liqiang. Operational Research of Air Defense Forces Combat [M].Press of Air Defense Command Academy, 2008.

[4] Zeng Xianzhao. New Methods of Military Optimization [M]. Press of Military Science, 2005.

[5] Ran Chengxin. "Research on the Detection Task programming Problem of Imaging Moving Target Based on Improved Genetic Algorithm" Journal of Astronautics,2010 